Pacific Journal of Mathematics

INTEGRALS WHICH ARE CONVEX FUNCTIONAL 


\title{
INTEGRALS WHICH ARE CONVEX FUNCTIONALS
}

\author{
R. T. Rockafellar
}

This paper examines numerical functionals defined on function spaces by means of integrals having certain convexity properties. The functionals are themselves convex, so they can be analysed in the light of the theory of conjugate convex functions, which has recently undergone extensive development. The results obtained are applicable to Orlicz space theory and in the study of various extremum problems in control theory and the calculus of variations.

In everything that follows, let $T$ denote a measure space with a $\sigma$-finite measure $d t$. Let $L$ be a particular real vector space of measurable functions $u$ from $T$ to $R^{n}$ (for a fixed $n$ ). For instance, one could take $L$ to be the space $L_{n}^{p}(T)$ consisting of all $R^{n}$-valued measurable functions $u$ on $T$ such that $\Phi_{p}(u)<+\infty$, where

$$
\Phi_{p}(u)=\int_{T} \varphi_{p}(u(t)) d t \quad \text { and } \varphi_{p}(x)=(1 / p)|x|^{p}, 1 \leqq p<+\infty
$$

with $|\cdot|$ denoting the Euclidean norm on $R^{n}$. No matter which $L$ is chosen, one can regard $\Phi_{p}$ as a functional from $L$ to $(-\infty,+\infty]$. Then $\Phi_{p}$ is convex, in consequence of the fact that the function $\phi_{p}$ is convex on $R^{n}$. (A function $F$ from a real vector space to $(-\infty,+\infty$ ] is said to be convex if

$$
F(\lambda x+(1-\lambda) y) \leqq \lambda F(x)+(1-\lambda) F(y)
$$

always holds when $0<\lambda<1$.) Notice that, if $\varphi_{\infty}$ is the convex function defined by

$$
\varphi_{\infty}(x)=\lim _{p \rightarrow \infty} \varphi_{p}(x)=\left\{\begin{array}{ccc}
0 & \text { if } & |x| \leqq 1 \\
+\infty & \text { if } & |x|>1,
\end{array}\right.
$$

the corresponding integral $\Phi_{\infty}(u)$ is finite if and only if $u$ belongs to the unit ball of the space $L_{n}^{\infty}(T)$ of essentially bounded measurable functions.

Here we propose to study a much broader class functionals than the $\Phi_{p}, 1 \leqq p \leqq \infty$. These functionals are of the form

$$
I_{f}(u)=\int_{T} f(t, u(t)) d t \quad \text { for } \quad u \in L
$$

where $f$ is a function from $T \times R^{n}$ to $(-\infty,+\infty]$, such that $f(t, x)$ is a convex function of $x \in R^{n}$ for each $t \in T$. Such a function $f$ we call a convex integrand for convenience.

As a preliminary task, we must come up with conditions on $f$ 
ensuring that various functions such as $f(t, u(t))$ be measurable in $t$. The well-known condition of Carathéodory is no help, because we do not want to assume that $f(t, x)$ is continuous in $x$. That would prevent us from considering most of the cases were $f$ can be infinity-valued. We have already encountered one such case, namely $f=\varphi_{\infty}$. Generally speaking, the device of allowing $f$ to have the value $+\infty$ has the effect of constraining $u(t)$ to lie in a certain convex subset of $R^{n}$, depending perhaps on $t$. Indeed, a necessary condition for $I_{f}(u)$ to be finite is that

$$
u(t) \in \operatorname{dom} f_{t} \quad \text { for almost all } t,
$$

where $f_{t}$ denotes the convex function $f_{t}(x)=f(t, x)$. (For any convex function $F$, the set of points where $F$ does not have the value $+\infty$ is a convex set, which we call the effective domain of $F$ and denote by $\operatorname{dom} F$.)

In order that $I_{f}(u)$ be an unambiguous number in $(-\infty,+\infty$, a further condition besides measurability, is usually needed, since $f(t, x)$ is not required to be nonnegative. The important thing, however, is that $I_{f}$ turns out to be a convex function on $L$ when it is well-defined.

The $\Phi_{p}$ have already been cited as examples of convex functionals of type $I_{f}$ which have received close attention from functional analysts. If the integrands $\varphi_{p}$ are generalized to those of the form $f(t, x)=$ $N(|x|)$ where $N$ is a finite nonnegative convex function on the real line such that $N(\lambda)>0$ for $\lambda>0$,

$$
\lim _{2 \uparrow 0} N(\lambda) / \lambda=0 \text { and } \lim _{\lambda \uparrow \infty} N(\lambda) / \lambda=\infty .
$$

one gets convex functionals $I_{f}$ defining generalized $L^{p}$ spaces, called Orlicz spaces. These spaces are very useful in dealing with integral equations. We refer the reader to [5] for an excellent account.

Possible applications along the lines suggested by the theory of Orlicz spaces are one motivation for looking at the convex functionals $I_{f}$ in the general case. Another motivation is that such functionals arise naturally in the calculus of variations. For example, suppose that $T=[0,1]$, with $d t$ as the ordinary Lebesgue measure. Regarding $R^{n}$ as $R^{k} \oplus R^{k}$, write each vector $x$ as a pair $(y, z)$, where $y$ and $z$ have $k$ components. Then $I_{f}$ may be interpreted as a functional defined for pairs of measurable functions from $[0,1]$ to $R^{k}$. Now let

$$
J(q)=I_{f}(q, \dot{q})=\int_{T} f(t, q(t), \dot{q}(t)) d t,
$$

where $q$ is a differentiable function from $[0,1]$ to $R_{k}$ (a curve) and $\dot{q}=d q / d t$. Inasmuch as differentiation is a linear operation, $J$ will be a convex function on the space of curves $q$. Problems which involve 
minimizing $J$ can hopefully be tackled therefore by convexity methods, such as the existence and duality theory in [11]. Note that infinite values of $f$ correspond to constraints on the values of $q(t)$ and $\dot{q}(t)$ for the curves $q$ such that $J(q)<+\infty$. Nonclassical convex constrained minimization problems of this sort abound in control theory. We plan elsewhere to take up applications of our results to such areas.

The main question treated in this paper is whether the conjugate of a convex functional $I_{f}$ is another such functional $I_{g}$. The question is significant, because the present theory of convex functions is so extensively concerned with conjugates. The notion of conjugacy, due to Fenchel [2], may be formulated in a general way as follows. Let $E$ and $E^{*}$ be real vector spaces, and let $\left\langle x, x^{*}\right\rangle$ be a (real) bilinear function of $x \in E$ and $x^{*} \in E^{*}$. Let $F$ be a proper convex function on $E$ (i.e. a convex function with values in $(-\infty,+\infty]$ which is not identically $+\infty$ ). The function $F^{*}$ on $E^{*}$ defined by

$$
F^{*}\left(x^{*}\right)=-\inf \left\{F(x)-\left\langle x, x^{*}\right\rangle \mid x \in E\right\}
$$

is called the conjugate of $F$ (with respect to the given pairing of $E$ and $E^{*}$ by $\left.\langle\cdot, \cdot\rangle\right)$. It is a convex function on $E^{*}$ with values in $(-\infty,+\infty]$. Furthermore, $F^{*}$ is always lower semi-continuous with respect to the weak topology induced on $E^{*}$ by $E$. (Lower semicontinuity means that the set $\left\{x^{*} \mid F^{*}\left(x^{*}\right) \leqq \mu\right\}$, which incidentally is always convex, is closed for every real $\mu$.) The conjugate of $F^{*}$ is in turn the function $F^{*}$ on $E$ defined by

$$
F^{* *}(x)=-\inf \left\{F^{*}\left(x^{*}\right)-\left\langle x, x^{*}\right\rangle \mid x^{*} \in E^{*}\right\} .
$$

In order that $F^{*}$ be proper and $F^{* *}=F$, it is necessary and sufficient that $F$ itself be lower semi-continuous with respect to the weak topology induced on $E$ by $E^{*}$. General proofs of these result are given in [1] and [6].

Two conjugacy contexts will mostly concern us here. In the first case, $E=E^{*}=R^{n}$ with $\left\langle x, x^{*}\right\rangle$ as the ordinary inner product. The weak topologies are then the ordinary topologies on $R^{n}$. In the second case we take $E=L$ and $E^{*}=L^{*}$, where $L^{*}$ is any space of $R^{n}$-valued measurable functions, such that the inner product $\left\langle u(t), u^{*}(t)\right\rangle$ is summable as a function of $t$ for every $u \in L$ and $u^{*} \in L^{*}$. The pairing is given by

$$
\left\langle u, u^{*}\right\rangle=\int_{T}\left\langle u(t), u^{*}(t)\right\rangle d t .
$$

Any topologies compatible with the duality between $E$ and $E^{*}$ could be invoked in place of the weak topologies, for instance the norm topologies if $E=E^{*}=L_{n}^{2}(T)$. 
Suppose that $f(t, x)$ is a convex integrand which is proper and lower semi-continuous in $x$ for each $t$. Define $f^{*}\left(t, x^{*}\right)$ by taking conjugates in $x$, i.e. $f_{t}^{*}=\left(f_{t}\right)^{*}$ for each $t$. Then, according to the results described above, $f^{*}$ is another convex integrand, proper and lower semi-continuous in its convex argument. We call it the integrand conjugate to $f$. The conjugate of the conjugate is the original integrand $f$. The principal fact brought out in this paper (Theorem 2) is that conjugate integrands $f$ and $f^{*}$ usually furnish conjugate functionals of $L$ and $L^{*}$. This generalizes the fact that $\varphi_{p}$ is conjugate to $\varphi_{q}$, and $\Phi_{p}$ on $L_{n}^{p}(T)$ is conjugate to $\Phi_{q}$ on $L_{n}^{q}(T)$ (with $\left.(1 / p)+1 / q\right)=1$ ). The resulting class of "best inequalities" of the type

$$
\left\langle u, u^{*}\right\rangle \leqq I_{f}(u)+I_{f^{*}}\left(u^{*}\right)
$$

is likewise a generalization of certain classical inequalities.

2. Normal integrands and measurability. Before we can proceed, we must established that various technical constructions result in functions which are measurable. To this end, some regularity conditions must be imposed. We shall call a convex integrand $f$ normal if $f(t, x)$ is proper and lower semi-continuous in $x$ for each $t$, and if further there exists a countable collection $U$ of measurable functions $u$ from $T$ to $R^{n}$ having the following properties:

(a) for each $u \in U, f(t, u(t))$ is measurable in $t$;

(b) for each $t, U_{t} \cap \operatorname{dom} f_{t}$ is dense in $\operatorname{dom} f_{t}$, where

$$
U_{t}=\{u(t) \mid u \in U\} \text {. }
$$

The latter conditions, which seem offhand to be rather complicated, are automatically satisfied in some notable cases, as we shall now indicate.

Lemma 1. Suppose $f(t, x)=F(x)$ for all $t$, where $F$ is a lower semi-continuous proper convex function on $R^{n}$. Then $f$ is a normal convex integrand.

Proof. Let $D$ be a countable dense subset of the effective domain of $F\left(=\operatorname{dom} f_{t}\right.$ for all $\left.t\right)$. (Such a $D$ exists, of course, because $\operatorname{dom} F$ is a nonempty convex set in $R^{n}$.) Let $U$ consist of the constant functions on $T$ with values in $D$. Then conditions (a) and (b) are satisfied in a trivial way.

Lemma 2. Suppose $f$ is a convex integrand such that $f(t, x)$ is measurable in $t$ for each fixed $x$, and such that, for each $t, f(t, x)$ is lower semi-continuous in $x$ and has interior points in its effective domain $\{x \mid f(t, x)<+\infty\}$. Then $f$ is a normal convex integrand. 
Proof. Let $D$ be a countable dense subset of $R^{n}$, and let $U$ be the constant functions with values in $D$. The measurability condition for normality is satisfied in virtue of the present measurability hypothesis. The density condition is satisfied, because $D$ has a dense intersection with the interior of $\operatorname{dom} f_{t}$, and $\operatorname{dom} f_{t}$ is the closure of its interior by convexity.

CoROLlary. Suppose $f$ is a convex integrand having only finite values, such that $f(t, x)$ is measurable in $t$ for each $x$. Then $f$ is a normal convex integrand.

Proof. Here $\operatorname{dom} f_{t}=R^{n}$ for every $t$. The lower semi-continuity of $f_{t}$ is then automatic, since a finite convex function on an open convex set in $R^{n}$ is always continuous.

An intermediate fact about the consequences of normality will now be deduced.

LeMma 3. Let $f$ be a normal convex integrand with conjugate $f^{*}$. Then, for every measurable function $u^{*}$ from $T$ to $R^{n}$, the function $f^{*}\left(t, u^{*}(t)\right)$ is measurable in $t$.

Proof. By definition,

$$
-f^{*}\left(t, u^{*}(t)\right)=\inf \left\{f(t, x)-\left\langle x, u^{*}(t)\right\rangle \mid x \in R^{n}\right\} .
$$

We shall show that, for each $t$, the infimum can actually be taken over $x \in U_{t}$ instead, where $U_{t}$ is the set in the definition of normality. Since $f(t, x)=+\infty$ for $x \notin \operatorname{dom} f_{t}$, the question is whether any value of $f(t, x)=\left\langle x, u^{*}(t)\right\rangle$ with $x \in \operatorname{dom} f_{t}$ can be approximated by one with $x \in U_{t} \cap \operatorname{dom} f_{t}$. Now $U_{t} \cap \operatorname{dom} f_{t}$ is dense in $\operatorname{dom} f_{t}$ by hypothesis. Furthermore $\operatorname{dom} f_{t}$, being a nonempty convex set, is the closure of its relative interior (its interior relative to the affine manifold it generates). The intersection of $U_{t}$ with this relative interior must be dense in $\operatorname{dom} f_{t}$. According to familiar results about lower semi-continuous convex functions (e.g. in [3], [13]), $f_{t}$ is continuous with respect to the relative interior of $\operatorname{dom} f_{t}$ and its values at relative boundary points can be obtained as limits of the relative interior values. Therefore the values of $f(t, x)$ for $x \in \operatorname{dom} f_{t}$ are limits of those for $U_{t} \cap \operatorname{dom} f_{t}$, as we wanted to show. The upshot is that

$$
-f^{*}\left(t, u^{*}(t)\right)=\inf \left\{f(t, u(t))-\left\langle u(t), u^{*}(t)\right\rangle \mid u \in U\right\} .
$$

This formula expresses $f^{*}\left(t, u^{*}(t)\right)$ as the pointwise infimum of a collection of functions on $T$. Each of the functions in the collection is measurable, in view of the hypotheses, and the collection is countable. 
The pointwise infimum is consequently another measurable function on $T$.

Moreau's proximation mappings, whose properties are elucidated in [9], will be very useful to us. Here is how they are defined. Let $F$ be any lower semi-continuous closed proper convex function on $R^{n}$. It can be proved that, for each $z \in R^{n}$, there exist unique vectors $x$ and $x^{*}$ such that

$$
z=x+x^{*} \text { and } F(x)+F^{*}\left(x^{*}\right)=\left\langle x, x^{*}\right\rangle .
$$

We write

$$
x=\operatorname{prox}(z \mid F) \text { and } x^{*}=\operatorname{prox}\left(z \mid F^{*}\right) .
$$

The mapping prox $(\cdot \mid F)$ from $R^{n}$ into itself is called the proximation associated with $F$. It is continuous (a metric contraction as a matter of fact), and its range is dense in $\operatorname{dom} F$. If $F$ is the indicator function of a closed convex set $K$ (in other words $F(x)=0$ when $x \in K$ and $F(x)=+\infty$ when $x \notin K)$, then prox $(z \mid F)$ is the point of $K$ nearest to $z$. In general, $\operatorname{prox}(z \mid F)$ is the unique $x$ for which

$$
F(x)+\frac{1}{2}|x-z|^{2}
$$

achieves its minimum.

LemMa 4. Let $f$ be a normal convex integrand. Let $z$ be a measurable function from $T$ to $R^{n}$. Then the functions prox $\left(z(t) \mid f_{t}\right)$ and prox $\left(z(t) \mid f_{t}^{*}\right)$ are measurable in $t$.

Proof. Set

$$
g(t, x)=f(t, x)+\frac{1}{2}|x-z(t)|^{2} .
$$

It is easily verified that $g$ is another normal convex integrand. We shall be concerned with the conjugate integrand $g^{*}\left(t, x^{*}\right)$. By Moreau's theory, $g_{t}^{*}$ is differentiable at 0 for each $t$, and $\nabla g_{t}^{*}(0)=\operatorname{prox}\left(z(t) \mid f_{t}\right)$. Now, for an arbitrary $a \in R^{n}$,

$$
\left\langle a, \nabla g_{t}^{*}(0)\right\rangle=\lim _{\lambda \downarrow 0}\left[g^{*}(t, \lambda a)-g^{*}(t, 0)\right] / \lambda .
$$

The difference quotient is a measurable function of $t$ for each $\lambda$ by Lemma 3 and the normality of $g$. The limit can be taken over a countable sequence in $\lambda$, so $\left\langle a, \nabla g_{t}^{*}(0)\right\rangle$ is measurable in $t$. It follows that $\operatorname{prox}\left(z(t) \mid f_{t}\right)$ is measurable in $t$, and likewise prox $\left(z(t) \mid f_{t}^{*}\right)$ because

$$
\operatorname{prox}\left(z(t) \mid f_{t}^{*}\right)=z(t)-\operatorname{prox}\left(z(t) \mid f_{t}\right)
$$


for every $t$.

We can now prove that normality is preserved when one passes to the conjugate.

LEMMA 5. If $f$ is a normal convex integrand, then $f^{*}$ is a normal convex integrand, too.

Proof. We already know from the theory of conjugates that $f^{*}\left(t, x^{*}\right)$ is a lower semi-continuous proper convex function of $x^{*}$ for each $t$. The problem is to produce a collection $U$ satisfying condition (b) of normality (with $f^{*}$ in place of $f$ ). Condition (a) will then hold by virtue of Lemma 3 . Let $D$ be any countable dense subset of $R^{n}$. Let $U$ consist of the functions of the form $u(t)=\operatorname{prox}\left(z \mid f_{t}^{*}\right)$ with $z$ ranging over $D$. Each $u \in U$ is measurable by Lemma 4 . The set $U_{t}$ is the image of $D$ under $\operatorname{prox}\left(\cdot \mid f_{t}^{*}\right)$. Since the proximation is continuous and its range is dense in $\operatorname{dom} f_{t}^{*}, U_{t}$ is dense in $\operatorname{dom} f_{t}^{*}$.

CoRollary. If $f$ is a normal convex integrand, then $f(t, u(t))$ is measurable in $t$ for every measurable function $u$ from $T$ to $R^{n}$.

Proof. This is immediate from Lemma 3, since $f_{t}=f_{t}^{* *}$.

Our final lemma guarantees the existence of enough measurable functions for one to minimize a normal convex integrand pointwise in a measurable fashion.

Lemma 6. Let $f$ be a normal convex integrand. Let $\alpha$ be a measurable real-valued function on $T$ such that

$$
\inf _{x} f(t, x)<\alpha(t) \quad \text { for every } t \text {. }
$$

Then there exists a measurable function $u$ from $T$ to $R^{n}$ such that

$$
f(t, u(t)) \leqq \alpha(t) \quad \text { for every } t \text {. }
$$

Proof. Set $K_{t}=\{x \mid f(t, x) \leqq \alpha(t)\}$ for each $t$. According to the general theory of convex functions on $R^{n}$, each $K_{t}$ is a nonempty closed convex subset of $\operatorname{dom} f_{t}$ having the same dimension as $\operatorname{dom} f_{t}$, inasmuch as $f_{t}(x)<\alpha(t)$ for at least one $x$. Therefore $U_{t} \cap K_{t}$ is dense in $K_{t}$, where $U_{t}$ is the set in the definition of the normality of $f$. Let $g(t, x)=0$ when $x \in K_{t}$ and $g(t, x)=+\infty$ when $x \notin K_{t}$. Evidently $g$ is another convex integrand satisfying the normality conditions with the same collection $U$ as invoked for $f$. Let $u(t)$ be the point of $K_{t}$ nearest to the origin, i.e.

$$
u(t)=\operatorname{prox}\left(0 \mid g_{t}\right) \text { for each } t .
$$


This $u$ is a measurable function by Lemma 4 (applied to $g$ ), and $f(t, u(t)) \leqq \alpha(t)$ by definition of $K_{t}$.

3. Conjugate convex integrals. The stage is now set for proving our chief results. We assume throughout that $L^{*}$ is a space of measurable functions paired with $L$ in the manner described in the introduction. (When $L$ is a Banach space, $L^{*}$ does not have to be its dual.)

THEOREM 1. Let $f$ be a normal convex integrand. Suppose there exists at least one $u^{*} \in L^{*}$ such that $f^{*}\left(t, u^{*}(t)\right)$ is a summable function of $t$. Then

$$
I_{f}(u)=\int_{T} f(t, u(t)) d t, \quad u \in L,
$$

is a well-defined convex function on $L$ with values in $(-\infty,+\infty]$.

Proof. The measurability prerequisite to considering $I_{f}$ is ensured by the corollary to Lemma 5 . Let $u^{*}$ be one of the functions in $L^{*}$ whose existence is provided for in the hypothesis. Since $f_{t}$ and $f_{t}^{*}$ are conjugate to each other

$$
f(t, u(t)) \geqq\left\langle u(t), u^{*}(t)\right\rangle-f^{*}\left(t, u^{*}(t)\right)
$$

for every $t$. The right side is a summable function of $t$ by the hypothesis. Thus there can be no question of $I_{f}(u)$ being $-\infty$ : either $f(t, u(t))$ is summable or its integral is unambiguously $+\infty$. As for the convexity of $I_{f}$, that is immediate from the inequality

$$
f(t, \lambda u(t)+(1-\lambda) v(t)) \leqq \lambda f(t, u(t))+(1-\lambda) f(t, v(t)),
$$

which holds for every $t$ when $0<\lambda<1$ by the convexity of $f_{t}$.

We shall say that $L$ is decomposable when it satisfies the following conditions:

(a) $L$ contains every bounded measurable function from $T$ to $R^{n}$ which vanishes outside a set of finite measure;

(b) if $u \in L$ and $E$ is a set of finite measure in $T$, then $L$ contains $\chi_{E} \cdot u$, where $\chi_{E}$ is the characteristic function of $E$.

These conditions guarantee that one can alter functions in $L$ arbitrarily in a bounded manner on sets of finite measure. (Subtract $\chi_{E} \cdot u$ from $u$, and then add any bounded measurable function vanishing outside $E$.) The first condition also implies that the functions in $L^{*}$ are summable on sets of finite measure. The $L_{n}^{p}(T)$ are examples of of spaces decomposable in this sense.

THEOREM 2. Suppose $L$ and $L^{*}$ are decomposable. Let $f$ be a 
normal convex integrand such that $f(t, u(t))$ is summable in $t$ for at least one $u \in L$, and $f^{*}\left(t, u^{*}(t)\right)$ is summable in $t$ for at least one $u^{*} \in L^{*}$. Then $I_{f}$ on $L$ and $I_{f^{*}}$ on $L^{*}$ are proper convex functions conjugate to each other.

Proof. $I_{f}$ and $I_{f^{*}}$ are well-defined and convex by Theorem 1 and Lemma 5, and they are proper by the hypothesis. For any $x^{*}$ in $R^{n}$ we have

$$
f(t, x)+f^{*}\left(t, x^{*}\right) \geqq\left\langle x, x^{*}\right\rangle
$$

by conjugacy. Hence, for any $u \in L$ and $u^{*} \in L^{*}$,

$$
\begin{gathered}
I_{f}(u)+I_{f^{*}}\left(u^{*}\right)=\int_{T} f(t, u(t)) d t+\int_{T} f^{*}\left(t, u^{*}(t)\right) d t \\
\geqq \int_{T}\left\langle u(t), u^{*}(t)\right\rangle d t=\left\langle u, u^{*}\right\rangle .
\end{gathered}
$$

It follows that

$$
\begin{aligned}
I_{f^{*}}\left(u^{*}\right) & \geqq \sup \left\{\left\langle u, u^{*}\right\rangle-I_{f}(u) \mid u \in L\right\} \\
& =-\inf \left\{I_{f}(u)-\left\langle u, u^{*}\right\rangle \mid u \in L\right\}=\left(I_{f}\right)^{*}\left(u^{*}\right) .
\end{aligned}
$$

Verification of the opposite inequality will establish that $I_{f^{*}}$ is the conjugate of $I_{f}$. Fix any $u^{*} \in L^{*}$ and any $\beta<I_{f *}\left(u^{*}\right)$. Select any real summable function $\mu$ on $T$ such that

$$
\mu(t)<f^{*}\left(t, u^{*}(t)\right) \text { for all } t, \text { and } \int_{T} \mu(t) d t>\beta \text {. }
$$

Since by conjugacy

$$
f^{*}\left(t, x^{*}\right)=-\inf \left\{f(t, x)-\left\langle x, x^{*}\right\rangle \mid x \in R^{n}\right\},
$$

we have

$$
-\mu(t)>\inf \left\{f(t, x)-\left\langle x, u^{*}(t)\right\rangle \mid x \in R^{n}\right\}
$$

for all $t$. We now apply Lemma 6 to $\alpha(t)=-\mu(t)$ and $g$, where

$$
g(t, x)=f(t, x)-\left\langle x, u^{*}(t)\right\rangle .
$$

(The normality of $f$ carries over to $g$.) The function $u$ we obtain from Lemma 6 satisfies

$$
-\mu(t) \geqq f(t, u(t))-\left\langle u(t), u^{*}(t)\right\rangle .
$$

Since $T$ is $\sigma$-finite by our underlying assumption, we can choose an increasing sequence of measurable sets $E_{k}$ of finite measure with union $T$, such that the $u(t)$ we have constructed is bounded in $t \in E_{k}$ for each $k$. Let $\bar{u}$ be any particular function in $L$ for which the in- 
tegrand in $I_{f}$ is summable. (Such a function exists by hypothesis.) For each $k$ let

$$
u_{k}(t)= \begin{cases}u(t) & \text { if } t \in E_{k} \\ \bar{u}(t) & \text { if } t \in E_{k}^{\prime}\end{cases}
$$

where $E_{k}^{\prime}$ denotes the complement of $E_{k}$ in $T$. These functions $u_{k}$ belong to $L$ by the decomposability hypothesis. For each $k$ we have

$$
\begin{gathered}
\int_{E_{k}} \mu(t) d t \leqq \int_{E_{k}}\left[\left\langle u(t), u^{*}(t)\right\rangle-f(t, u(t))\right] d t \\
=\left\langle u_{k}, u^{*}\right\rangle-I_{f}\left(u_{k}\right)-\int_{E_{k}^{\prime}}\left[\left\langle\bar{u}(t), u^{*}(t)\right\rangle-f(t, \bar{u}(t))\right] d t .
\end{gathered}
$$

The boundedness assumption on $E_{k}$ is used here to ensure that $\left\langle u_{k}(t), u^{*}(t)\right\rangle$ be summable, so that

$$
\int_{T}\left[\left\langle u_{k}(t), u^{*}(t)\right\rangle-f\left(t, u_{k}(t)\right)\right] d t=\left\langle u_{k}, u^{*}\right\rangle-I_{f}\left(u_{k}\right)
$$

unambiguously. The integral over $E_{k}^{\prime}$ in the calculation above can be made arbitrarily small by choosing $k$ sufficiently large. On the other hand

$$
\lim _{k \rightarrow \infty} \int_{E_{k}} \mu(t) d t=\int_{T} \mu(t) d t>\beta
$$

by our assumptions. Thus

$$
\left\langle u_{k}, u^{*}\right\rangle-I_{f}\left(u_{k}\right)>\beta
$$

when $k$ is large, implying that $\left(I_{f}\right)^{*}\left(u^{*}\right)>\beta$. Inasmuch as $\beta$ was any number less than $I_{f^{*}}$, we may now conclude that $I_{f^{*}}\left(u^{*}\right)=\left(I_{f}\right)^{*}\left(u^{*}\right)$. The fact that $I_{f}=\left(I_{f^{*}}\right)^{*}$ follows dually.

CoRollary. Suppose that $L$ and $L^{*}$ are decomposable, and that $T$ is of finite measure. Let $f$ be of the form $f(t, x)=F(x)$, where $F$ is a lower semi-continuous proper convex function on $R^{n}$. Then $I_{f}$ on $L$ and $I_{f^{*}}$ on $L^{*}$ are conjugate to each other.

Proof. Such an $f$ is normal by Lemma 1. The existence of summable function for $I_{f}$ and $I_{f^{*}}$ is elementary in this case. Namely, take any $x$ for which $F(x)$ is finite, and let $u$ be the constant function whose sole value is $x$. Since $T$ is of finite measure, $u$ is summable. By decomposability (b), $u \in L$. Similary for $I_{f^{*}}$. The hypothesis of Theorem 2 is therefore satisfied.

The next theorem furnishes a different way of establishing the 
conjugacy of $I_{f^{*}}$ and $I_{f}$ in certain situations. It also yields a continuity property.

Theorem 3. Let $T$ be of finite measure. Suppose that $L^{*}$ is decomposable, and that $L$ is actually $L_{n}^{\infty}(T)$. Let $f$ be a normal convex integrand satisfying the following condition: there exists some $a \in L$ and $\varepsilon>0$ such that, for each $x \in R^{n}$ with $|x|<\varepsilon$, the function $f(t, a(t)+x)$ is finite and bounded in $t$. Then $I_{f}$ on $L$ and $I_{f^{*}}$ on $L^{*}$ are convex functions conjugate to each other. Moreover, $I_{f}$ is continuous at $a$ in the norm topology of $L=L_{n}^{\infty}(T)$.

Proof. Replacing $f$ by $g$ if necessary, where

$$
g(t, x)=f(t, a(t)+x)-f(t, a(t))
$$

(evidently another normal convex integrand), we can reduce everything to the case where $a(t) \equiv 0$ and $f(t, 0) \equiv 0$. Then $I_{f}(0)=0$. We must show that $I_{f}$ is norm-continuous at 0 , and that $f^{*}\left(t, u^{*}(t)\right)$ is summable in $t$ for some $u^{*} \in L^{*}$. The conjugacy of $I_{f}$ and $I_{f^{*}}$ will then follow from the last theorem. Define

$$
F(x)=\sup \{f(t, x) \mid t \in T\} .
$$

As a pointwise supremum of lower semi-continuous convex functions on $R^{n}, F$ is itself lower semi-continuous and convex. By hypothesis, $F(x)$ is finite on the open convex set $\{x|| x \mid<\varepsilon\}$. As is well-known a finite convex function on a finite-dimensional open convex set is automatically continuous. Hence $F$ is continuous when $|x|<\varepsilon$. Fix a positive $\delta$ less than $\varepsilon$, and let

$$
k=\max \{F(x)|| x \mid \leqq \delta\}<+\infty .
$$

Now $F(0)=0$, so that we have $F(x) \leqq(k / \delta)|x|$ when $|x| \leqq \delta$ by convexity. (Consider the values of $F$ along the line segment from 0 to $\alpha x$, where $\alpha=\delta /|x|$.) Hence for every $t$

$$
f(t, x) \leqq(k / \delta)|x| \text { when }|x| \leqq \delta .
$$

This inequality also implies that $f(t, x) \geqq-(k / \delta)|x|$ for every $x$. (To verify this, one expresses 0 as a convex combination of $x$ and $\mu x$, where $\mu=-\delta /|x|$, namely $0=\lambda x+(1-\lambda) \mu x$ with $\lambda=-\mu /(1-\mu)$. Then by the convexity of $f$

$$
\begin{aligned}
0=f(t, 0) & \leqq \lambda f(t, x)+(1-\lambda) f(t, \mu x) \\
& \leqq \lambda f(t, x)+(1-\lambda)(k / \delta)|\mu x| \\
& =-(\mu /(1-\mu))[f(t, x)+(k / \delta)|x|] .
\end{aligned}
$$

The first inequality has been applied here to $\mu x$, which is permissible 
because $|\mu x|=\delta$ by the choice of $\mu$. One concludes that $f(t, x)+$ $(k / \delta)|x|$ is always non-negative.) In particular, therefore

$$
|f(t, x)| \leqq(k / \delta)|x| \text { when }|x| \leqq \delta .
$$

If $u \in L$ satisfies $\|u\| \leqq \delta$, where $\|\cdot\|$ is the $L_{n}^{\infty}(T)$ norm, then

$$
\int_{T}|f(t, u(t))| d t \leqq(k / \delta)\|u\| \text { meas } T<+\infty .
$$

Thus $I_{f}(u)$ is well-defined when $\|u\| \leqq \delta$, and it approaches $0=I_{f}(0)$ as $\|u\|$ approaches 0 . This establishes the continuity. We must still construct a $u^{*} \in L^{*}$ for which $f^{*}\left(t, u^{*}(t)\right)$ is summable. It suffices to find such a $u^{*}$ in $L_{n}^{\infty}(T)$, for $L^{*}$ contains $L_{n}^{\infty}(T)$ in consequence of the hypothesis that $L^{*}$ is decomposable and $T$ is of finite measure. Let

$$
\bar{u}(t)=\operatorname{prox}\left(0 \mid f_{t}\right) \text {. }
$$

The measurability of $\bar{u}$ is asserted by Lemma 4 . For each $t, \bar{u}(t)$ is the point which minimizes $f(t, x)+1 / 2|x|^{2}$ on $R^{n}$. Since the minimand vanishes at $x=0$,

$$
0 \geqq f(t, \bar{u}(t))+\frac{1}{2}|\bar{u}(t)|^{2} \geqq-(k / \delta)|\bar{u}(t)|+\frac{1}{2}|\bar{u}(t)|^{2} .
$$

It follows that $|\bar{u}(t)| \leqq 2 k / \delta$ for all $t$, so $\bar{u} \in L_{n}^{\infty}(T)$. It now follows further that

$$
0 \geqq f(t, \bar{u}(t)) \geqq-(k / \delta)|\bar{u}(t)| \geqq-2(k / \delta)^{2},
$$

so $f(t, \bar{u}(t))$ is bounded in $t$ (and hence summable). Now take

$$
u^{*}(t)=-\bar{u}(t)=0-\operatorname{prox}\left(0 \mid f_{t}\right)=\operatorname{prox}\left(0 \mid f_{t}^{*}\right) .
$$

Again $u^{*} \in L_{n}^{\infty}(T)$. According to the basic property of proximations,

$$
f(t, \bar{u}(t))+f^{*}\left(t, u^{*}(t)\right)=\left\langle\bar{u}(t), u^{*}(t)\right\rangle
$$

for every $t$. The first and last terms in this equation yield summable functions, so we can conclude that $f^{*}\left(t, u^{*}(t)\right)$ is summable, too.

THEOREM 4. Let $T$ be of finite measure. Let $f(t, x)$ be a finite convex function of $x$ for each $t$ and a bounded measurable function of $t$ for each $x$. Then $I_{f}$ is a well-defined finite convex function on $L_{n}^{\infty}(T)$ which is everywhere continuous with respect to the uniform norm. Moreover, the conjugate $\left(I_{f}\right)^{*}$ of $I_{f}$ on $L_{n}^{\infty}(T)^{*}$, the space of all linear function as on $L_{n}^{\infty}(T)$ continuous with respect to the uniform norm, is given by $I_{f *}$ in the following sense: if $v \in L_{n}^{\infty}(T)^{*}$ is of the form 


$$
v(u)=\int_{T}\left\langle u(t), u^{*}(t)\right\rangle d t, \quad u^{*} \in L_{n}^{1}(T),
$$

one has $\left(I_{f}\right)^{*}(v)=I_{f *}(u)$, whereas otherwise one has $\left(I_{f}\right)^{*}(v)=+\infty$.

Proof. We note that $f$ is a normal convex integrand by the corollary to Lemma 2. The finiteness and continuity of $I_{f}$ are asserted by Theorem 3. Fix any $v \in L_{n}^{\infty}(T)^{*}$ such that $\left(I_{f}\right)^{*}(v)<+\infty$. We shall show that $v$ corresponds to some $u^{*} \in L_{n}^{1}(T)$ as above, whence it will follow from Theorem 3 that

$$
\left(I_{f}\right)^{*}(v)=\sup _{u}\left\{v(u)-I_{f}(u)\right\}=I_{f *}\left(u^{*}\right) .
$$

For each measurable $E \subset T$, let $\mu(E)$ denote the unique vector in $R^{n}$ such that

$$
\langle x, \mu(E)\rangle=v\left(x \cdot \chi_{E}\right) \text { for every } x \in R^{n},
$$

where $x \cdot \chi_{E}$ is the function which has the value $x$ on $E$ but the value 0 elsewhere on $T$. Then $\mu$ is a finitely additive set function. We have

$$
\begin{aligned}
& \langle x, \mu(E)\rangle \leqq I_{f}\left(x \cdot \chi_{E}\right)+\left(I_{f}\right)^{*}(v) \\
& \quad=\int_{E} f(t, x) d t+\int_{T / E} f(t, 0) d t+\left(I_{f}\right)^{*}(v) \\
& \quad \leqq F(x) \text { meas } E+\alpha,
\end{aligned}
$$

where

$$
\begin{aligned}
F(x) & =\sup \{f(t, x) \mid t \in T\}<+\infty, \\
\alpha & =\max \{0, F(0) \text { meas } T\}+\left(I_{f}\right)^{*}(v)<+\infty .
\end{aligned}
$$

The function $F$ is convex, and hence continuous, so that the quantity

$$
k(r)=\sup \{F(x)|| x \mid \leqq r\}
$$

is finite for every $r>0$. For every measurable $E \subset T$ and every $r>0$, we have

$$
\begin{aligned}
r|\mu(E)| & =\sup \{<x, \mu(E)>|| x \mid \leqq r\} \\
& \leqq k(r) \text { meas } E+\alpha<+\infty .
\end{aligned}
$$

It follows that, given any $\varepsilon>0$, there exists a $\delta>0$ such that meas $E<\delta$ implies $|\mu(E)|<\varepsilon$. Thus $\mu$ is absolutely continuous with respect to $d t$, and $\mu$ must be countably additive. By the RadonNikodym Theorem, there exists some $u^{*} \in L_{n}^{1}(T)$ such that

$$
\int_{E}\left\langle x, u^{*}(t)\right\rangle d t=\langle x, \mu(E)\rangle=v\left(x \cdot \chi_{E}\right)
$$

for every $x \in R^{n}$ and every measurable $E$. The formula 


$$
v(u)=\int_{T}\left\langle u(t), u^{*}(t)\right\rangle d t
$$

then holds for every $u$ which is a linear combination of functions of the form $x \cdot \chi_{E}$, and since such linear combinations are dense in $L_{n}^{\infty}(T)$ the formula must actually hold for every $u \in L_{n}^{\infty}(T)$ by continuity.

COROLLARY 1. Under the hypothesis of Theorem 4, the convex set

$$
\left\{u^{*} \in L_{n}^{1}(T) \mid\left(I_{f *}\right)\left(u^{*}\right)+\left\langle\alpha, u^{*}\right\rangle+\alpha \leqq 0\right\}
$$

is weakly compact (with respect to the pairing between $L_{n}^{1}(T)$ and $L_{n}^{\infty}(T)$ ) for any $a \in L_{n}^{\infty}(T)$ and any real number $\alpha$.

Proof. Since $I_{f}$ norm-continuous, the set

$$
\left\{v \in L_{n}^{\infty}(T)^{*} \mid\left(I_{f}\right)^{*}(v)+v(a)+\alpha \leqq 0\right\}
$$

is weak* compact in $L_{n}^{\infty}(T)^{*}$ for any $a$ and $\alpha$, according to a theorem proved independently by Moreau [7] and the author [12, Theorem 7A].

Corollary 2. Let $D$ be a subspace of $L_{n}^{\infty}(T)$ supplied with a locally convex topology at least as strong os the uniform norm topology, and let $D^{*}$ be the space of continuous linear functionals on $D$. Suppose that no nonzero linear functional on $L_{n}^{\infty}(T)$ of the form

$$
u \rightarrow \int_{T}\left\langle u(t), u^{*}(t)\right\rangle d t, \quad u^{*} \in L_{n}^{1}(T),
$$

vanishes throughout $D$. Then, under the hypothesis of Theorem 4, $I_{f}$ is a continuous finite convex function on $D$, and the conjugate $\left(I_{f}\right)^{*}$ of $I_{f}$ on $D^{*}$ is given by $I_{f *}$, in the sense that if $v \in D^{*}$ corresponds to some $u^{*} \in L_{n}^{1}(T)$ as above one has $\left(I_{f}\right)^{*}(v)=I_{f *}\left(u^{*}\right)$, whereas otherwise $\left(I_{f}\right)^{*}(v)=+\infty$.

Proof. Let $J$ be the convex functional on $D^{*}$ such that $J(v)=$ $I_{f *}\left(u^{*}\right)$ if $v$ corresponds to a $u^{*} \in L_{n}^{1}(T)$, whereas otherwise $J(v)=+\infty$. This $J$ is well-defined, in view of the hypothesis about linear functionals which vanish on $D$, and the conjugate of $J$ on $D$ with respect to the natural pairing of $D$ and $D^{*}$, is just the restriction of $I_{f}$ to $D$. By Corollary 1, the convex sets

$$
\left\{v \in D^{*} \mid J(v) \leqq \mu\right\}, \quad \mu \in R,
$$

are compact in the weak topology on $D^{*}$ induced by $D$, so that $J$ is lower semi-continuous in this topology. It follows that $J=J^{* *}=\left(I_{f}\right)^{*}$.

REMARK. Corollary 2 is applicable, of course, to various situations where $T$ has topological or differentiable structure, and $D$ is a space 
of continuous or differentiable functions on $T$ (with $D^{*}$ a corresponding space of measures of distributions).

\section{REFERENCES}

1. A. Brondsted, Conjugate convex functions in topological vector spaces, Mat.-fys. Medd. Dansk. Vid. Selsk. 34 (1964).

2. W. Fenchel, On conjugate convex functions, Canad. J. Math. 1 (1949), 73-77.

3. Convex cones, sets and functions, lecture notes, Princeton University, 1953.

4. P. Halmos, Measure Theory, van Nostrand, Princeton, 1950.

5. M. A. Krasnosel'skii and Ya. B. Rutickii, Convex Functions and Orlicz Spaces, Noordhoff, Groningen, 1961.

6. J.-J. Moreau, Fonctions convexes en dualité, Séminaire de Mathématiques de la Faculté des Sciences de Montpellier, no. 1, 1962.

7. - Sur la fonction polaire d'une fonction semi-continue supériurement, C. R. Acad. Sci 258 (1964), 1128-1130.

8. Les liaisons unilaterals et le principe de Gauss, C. R. Acad. Sci. 256 (1963), 871-874.

9. - Proximité et dualité dans un espace hilbertien, Bull. Soc. Math. France 93 (1965), 273-299.

10. L. S. Pontryagin, V. G. Boltyanskii, R. V. Gamkrelidze and E. F. Mishchenko, The Mathematical Theory of Optimal Processes, Interscience, New York, 1962.

11. R. T. Rockafellar, Duality and stability in extemum problems involving convex functions, Pacific J. Math. 21 (1967), 167-187.

12. Level sets and continuity of conjugate convex functions Trans. Amer. Math. Soc. 123 (1966), 46-63.

13. - Convex analysis, lecture notes, Princeton University, 1966.

Received August 17, 1966. This work was supported by the Air Force Office of Scientific Research under Grant 1165-66 at Princeton University.

UNIVERSITY OF WASHINGTON 



\section{PACIFIC JOURNAL OF MATHEMATICS}

\section{EDITORS}

\author{
H. ROYDEN \\ Stanford University \\ Stanford, California
}

\author{
J. P. JANS \\ University of Washington \\ Seattle, Washington 98105
}

J. DugundJI

Department of Mathematics

Rice University

Houston, Texas 77001

RICHARD ARENS

University of California

Los Angeles, California 90024

\section{ASSOCIATE EDITORS}
E. F. BECKENBACH
B. H. NeumanN
F. WOLF
K. YOSIDA

\section{SUPPORTING INSTITUTIONS}

\author{
UNIVERSITY OF BRITISH COLUMBIA \\ CALIFORNIA INSTITUTE OF TECHNOLOGY \\ UNIVERSITY OF CALIFORNIA \\ MONTANA STATE UNIVERSITY \\ UNIVERSITY OF NEVADA \\ NEW MEXICO STATE UNIVERSITY \\ OREGON STATE UNIVERSITY \\ UNIVERSITY OF OREGON \\ OSAKA UNIVERSITY \\ UNIVERSITY OF SOUTHERN CALIFORNIA
}

\author{
STANFORD UNIVERSITY \\ UNIVERSITY OF TOKYO \\ UNIVERSITY OF UTAH \\ WASHINGTON STATE UNIVERSITY \\ UNIVERSITY OF WASHINGTON \\ AMERICAN MATHEMATICAL SOCIETY \\ CHEVRON RESEARCH CORPORATION \\ TRW SYSTEMS \\ NAVAL ORDNANCE TEST STATION
}

\footnotetext{
Mathematical papers intended for publication in the Pacific Journal of Mathematics should be in typed form or offset-reproduced, double spaced with large margins. Underline Greek letters in red, German in green, and script in blue. The first paragraph or two must be capable of being used separately as a synopsis of the entire paper. It should not contain references to the bibliography. Manuscripts may be sent to any one of the four editors. All other communications to the editors should be addressed to the managing editor, Richard Arens, University of California, Los Angeles, California 90024.

Each author of each article receives 50 reprints free of charge; additional copies may be obtained at cost in multiples of 50 .

The Pacific Journal of Mathematics is published monthly. Effective with Volume 16 the price per volume (3 numbers) is $\$ 8.00$; single issues, $\$ 3.00$. Special price for current issues to individual faculty members of supporting institutions and to individual members of the American Mathematical Society: $\$ 4.00$ per volume; single issues $\$ 1.50$. Back numbers are available.

Subscriptions, orders for back numbers, and changes of address should be sent to Pacific Journal of Mathematics, 103 Highland Boulevard, Berkeley 8, California.

Printed at Kokusai Bunken Insatsusha (International Academic Printing Co., Ltd.), 7-17, Fujimi 2-chome, Chiyoda-ku, Tokyo, Japan.

PUBLISHED BY PACIFIC JOURNAL OF MATHEMATICS, A NON-PROFIT CORPORATION

The Supporting Institutions listed above contribute to the cost of publication of this Journal, but they are not owners of publishers and have no responsibility for its content or policies.
} 


\section{Pacific Journal of Mathematics}

\section{Vol. 24, No. $3 \quad$ July, 1968}

Duane W. Bailey, On symmetry in certain group algebras ............ 413

Lawrence Peter Belluce and Surender Kumar Jain, Prime rings with a one-sided ideal satisfying a polynomial identity ................ 421

L. Carlitz, A note on certain biorthogonal polynomials ............. 425

Charles O. Christenson and Richard Paul Osborne, Pointlike subsets of a manifold ......................................... 431

Russell James Egbert, Products and quotients of probabilistic metric

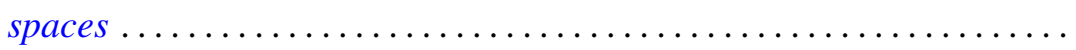

Moses Glasner, Richard Emanuel Katz and Mitsuru Nakai, Bisection into small annuli ..................................... 457

Karl Edwin Gustafson, A note on left multiplication of semigroup

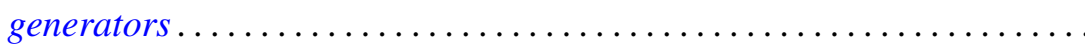

I. Martin (Irving) Isaacs and Donald Steven Passman, A characterization of groups in terms of the degrees of their characters. II ............. 467

Howard Wilson Lambert and Richard Benjamin Sher, Point-like

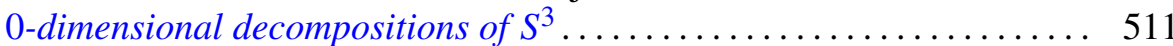

Oscar Tivis Nelson, Subdirect decompositions of lattices of width two ..... 519

Ralph Tyrrell Rockafellar, Integrals which are convex functionals . . . . . . . 525

James McLean Sloss, Reflection laws of systems of second order elliptic differential equations in two independent variables with constant coefficients ...

Bui An Ton, Nonlinear elliptic convolution equations of Wiener-Hopf type in a bounded region

Daniel Eliot Wulbert, Some complemented function spaces in $C(X)$

Zvi Ziegler, On the characterization of measures of the cone dual to a generalized convexity cone. 